Paper No. $249-9$

\title{
Role of Multi-slip on Plastic Behaviours of Polycrystalline Metals*
}

\author{
By Masataka TOKUDA** and Hideyuki $\mathrm{KATOH}^{* * *}$
}

\begin{abstract}
In this work, the plastic behaviours of polycrystalline metals in two typical examles of complex loading conditions are computationally analyzed by using a simplified mechanical model of polycrystal in the manner of a crystal plasticity type. The first example is a deformation along a strain path with a corner, and the second one is a so-called mechanical ratcheting deformation. The variations of microscopic stress in some single crystal components in the polycrystal model are discussed in detail, and then the relation between the macroscopic stress and microscopic stress is clarified. Especially, it is confirmed through the discussions that the multi-slip in each crystal component is an important mechanism related with some macroscopic features of polycrystalline metals under such complex loading conditions observed in the experiments.
\end{abstract}

Key Words : Strength of Material, Plasticity, Polycrystalline Metal, Crystal Plasticity, Constitutive Equation, Complex Loading, Internal Stress, Multi-Slip

\section{Introduction}

In the previous paper(1), plastic deformation mechanisms of polycrystalline metals under complex loading (non-radial, multi-axial loading) conditions were discussed by using a simple but significant mechanical model of polycrystal derived from a view point of crystal plasticity(2). It was suggested from the discussion that an occurence of multi-slip state $^{(3-5)}$ due to an increase of internal stress (a difference between a stress of single crystal component and an averaged stress of polycrystal) may be closely related with fundamental mechanisms causing deformation history effects observed in systematic and precise experiments under complex loading conditons (til. $^{\circ}$

In the first part of this paper, we will discuss, from a multi-slip point of view, several interesting phenomena in polycrystal deformed along a set of twosegment strain paths which are typical examples of complex deformation paths, on the basis of some computational results obtained by using the polycrystal model. In the second part, we pick up a mechanical ratcheting phenomenon as an example of more complex and severe loading process $^{(6-10)}$. When polycrystalline metals are subjected to a comparatively small stationary stress together with a cyclic strain or stress (which exceeds the yield limit) in a direction different from that

* Received 6th April, 1984

* Associate Prof., Department of Mechanical Engineering, Mie University, kamihama, Tsu 514, Japan

*** Engineer, Nippon Denso CoLtd., Showa, Kariya 448, Japan of stationary stress, a permanent strain (so-called ratcheting strain) is accumulated in the direction along the small stationary stress. This phenomenon is called a mechanical ratcheting. A typical example is a plastic behaviour of a thinwalled tubular specimen subjected to a combined load of a small stationary torsion and a cyclic tension-compression beyond the yield limit. In this case, the torsional strain is accumulated in each cycle. In this way, some elements of machine parts subjected to such loadings may lose their functions owing to the excess deformation along the stationary load which is much smaller than the amplitude of cyclic loading.

The ratcheting strain - the number of cycles curve has a similar shape to a creep strain-time curve ${ }^{(6,8)}$ which has a transient creep stage and a stationary creep stage. Moreover, when we unload the stationary load after the ratcheting strain exceeds a certain value while we keep the cyclic loading, the accumulated ratcheting strain tends to decrease in a similar way to a recovery phenomenon of creep strain observed after the unloading. This phenomenon is called a recovery of mechanical ratcheting strain. In this paper, such fundamental features of mechanical ratcheting are discussed on the basis of computationally analyzed results. It is found out from the discussions that mechanisms of these phenomena are also related with the multi-slip in each single crystal component.

The clarifications of such deformation mechanisms may be helpful to proper understanding of the complex plastic behaviours of polycrystals and to formulation of reasonably precise constitutive equations ${ }^{111-13]}$ incorporating such features, especially under multi-axial conditions. 
2. Mechanical Models of Single Crystal and Polycrystal

The mechanical models of single crystal and polycrystal used here are ones simplified as much as possible without any loss of fundamental mechanisms of plastic deformations in polycrystalline metals, which were proposed in the previous paper?. Here they are explained briefly.

The single crystal model has only one slip plane and three pairs of easy slip directions apart $60^{\circ}$ from each other on the plane [see Fig. 1(a)]. That is, three pairs of slip systems are considered in a grain, by counting a slip system with opposite slip direction as another slip system. An initiation of slip is supposed to be controlled by Schmid law. That is, when a resolved shear stress on a slip direction and a slip plane of slip system reaches a certain value (critical resolved shear stress), the system yields a plastic shear (slip) strain. The critical resolved shear stress is assumed to foll 17 ow the linearized Taylor hardening

$$
\tau^{(1)}=\tau^{(2)}=\cdots=\tau^{(N)}=\tau_{0}+H \sum_{i=1}^{N} \gamma^{(i)}
$$

where $\tau^{(i)}$ and $\gamma^{(i)}$ are the critical resolved shear stress and the slip strain of the $i-t h$ slip system, respectively, and $N$ is the number of slip systems in a grain ( $N=6$ for our model), $H$ is a hardening parameter, $\tau_{0}$ is an initial critical resolved shear stress. The mechanical model of single crystal is plastically anisotropic because it has a discrete distribution of slip systems, but it is assumed to be elastically isotropic and follow the linearized Hooke's law, for simplicity. The polycrystal model is an aggregate of the idealized single crystal models whose slip planes are placed in an $x-y$ plane of a reference cordinate system xyz of Descartes type and whose slip directions are arranged to be distributed uniformly in the $x-y$ plane in the averaged meaning over all grains of the polycrystalline model. Interactions among grains in such a two-dimensional polycrystal model are incorporated by using the self-consistent scheme proposed by Kröner.16) When the polycrystal model is deformed along a two-dimensional strain path described in terms of (macroscopic, i.e., averaged) shear strain components $e_{2 x}$ and $e_{z y}$, two microscopic stress components $\tau_{z x}^{*}$ and $\tau_{z y}^{*}$ and two microscopic strain components $e_{z x}^{*}$ and $e_{z y}^{*}$ occur in each single crystal components. Then, macroscopic stress responses $\tau_{z x}$ and $\tau_{z y}$ can be obtained by averaging $\tau_{z x}^{*}$ and $\tau_{z y}^{*}$, respectively, over all single crystal components in the polycrystal.

According to the assumption of selfconsistent scheme, a macroscopic stress state of a single crystal component with an orientation $\alpha$ (see Fig. $1(\mathrm{a})$ ) is related to a macroscopic stress state in the following vector form;

$$
\vec{s}^{*}(\alpha)=\bar{s}-\lambda G\left[\bar{e}_{p}^{*}(\alpha)-\bar{e}_{p}\right]
$$

where $\bar{s}=\left(\sqrt{3} \tau_{z x}, \sqrt{3} \tau_{z y}\right), \quad \bar{e}_{p}=\left(2 e_{x x}^{p} / \sqrt{3}, 2 e_{x y}^{p} / \sqrt{3}\right)$, $\bar{s}^{*}(\alpha)=\left(\sqrt{3} \tau_{z x}^{*}(\alpha), \sqrt{3} \tau_{z y}^{*}(\alpha)\right), \bar{e}_{p}^{*}=\left(2 e_{z x}^{* p}(\alpha) / \sqrt{3}, 2 e_{z y}^{* *}(\alpha)\right.$ $(\sqrt{3})$. This type of vector representation comes from the generalized vector spaces of stress and strain deviators proposed by Ilyushill. ${ }^{-191} e_{2 x}^{p}, e_{z y}^{p}, e_{z x}^{* p}$ and $e_{z y}^{* p}$ are plastic parts of $e_{z x}, e_{z y}, e_{z x}^{*}$ and $e_{z y}^{*}$, respectively, and $G$ is the shear modulus. The parameter $\lambda$ is a material constant expressed in terms of Poisson's ratio $\nu$, as follows.

$$
\lambda=2(7-5 \nu) /\{15(1-\nu)\}
$$

The vector $\bar{s}-\bar{s}^{*}(\alpha)\left[\equiv s_{l}(\alpha)\right]$ denotes a difference between a stress state of single crystal component with orientation $\alpha$ and a macroscopic stress state of polycrystal. The difference vector $\bar{s}_{t}(\alpha)$ is called an internal stress and related with a difference between the corresponding two plastic strain vectors, as seen in Eq. (2). The material constants of polycrystal model explained here are $G, \tau_{0}, H$ and $\lambda($ or $\nu)$.

By using the simple mechanical model of polycrystal,

[a] stress responses along a set of twosegment strain paths, and

[b] mechanical ratcheting phenomenon are computationally analyzed, and micromechanisms of plastic deformation of polycrystal under such complex loading conditions are discussed from a multi-slip point of view.

Lin's strain-constant assumption is used instead of Kröner's self-consistent assumption in the analysis of [a], because we can save the computation time and evaluate easily the obtained results from the mechanical points of view, when the strain path is given. Lin's assumption is a kind of approximation of Kröner's assumption (the parameter $\lambda$ in Eq. (2) equals to 2 in Lin's assumption). On the other hand, Lin's approximation is not used for the analysis of [b] because the advantage on computation time disappears in this case. The difference between these two assumptions is not substantial in the qualitative aspect and thus, the generality of discussions described may not be lost. An example of direct comparison between computational results obtained by the two assumptions given in Fig. 6 and explained later.

3. Stress Responses for Two-segment Strain Paths and Multislip

As shown in Fig. 1(a), three pairs of systems are labeled 1-6, respectively and the orientation of a single crystal component is denoted by an angle $\alpha$ between the $\mathrm{x}$-axis and the slip direction of the slip system 1. The value of $\alpha$ can be limited to $-30^{\circ}<\alpha \leqq 60^{\circ}$ without any loss 
of generality because of a symmetry of the single crystal model. A stress plane $\left[\sqrt{3} \tau_{z x}^{*}, \sqrt{3} \tau_{z y}^{*}\right]$ is convenient to show plastic properties of single crystal model. For example, in this plane, an initial yield surface of single crystal model is shown by a regular hexagon whose center coincides with the origin 0 of the plane and the distance between each edge and the center 0 equals to the value of $\sqrt{3} \tau_{0}\left(\tau_{0}\right.$; initial critical resolved shear stress),according to the Schmid law. Moreover, by overlapping a plane $\left[\sqrt{3} \tau_{z x}, \sqrt{3} \tau_{z y}\right]$ on the plane $\left[\sqrt{3} \tau_{z x}^{*}, \sqrt{3} \tau_{z y}^{*}\right]$ in a co-axial manner, we can illustrate the vectors $\bar{s}, \vec{s}^{*}$ and $\bar{s}_{t}$ $\left(\equiv \bar{s}^{*}-\bar{s}\right)$ simultaneously on the plane and thus this plane is helpful for us to discuss the relation between macroscopic and microscopic behaviours of polycrystal. A two-dimensional strain path composed of variations of $e_{z x}$ and $e_{z y}$ can be illustrated in a strain plane $\left[2 e_{z x} / \sqrt{3}, 2 e_{z y} /\right.$ $\sqrt{3}$, which is a conjugate plane of $\left[\sqrt{3} \tau_{z x}, \sqrt{3} \tau_{z y}\right]$.

Now, let's discuss about microscopic stresses $\vec{s}^{*}$ in the single crystal components and macroscopic stresses $\bar{s}$ in the polycrystal model deformed along some twosegment strain paths in the strain plane $\left[2 e_{z x} / \sqrt{3}, 2 e_{z y} / \sqrt{3}\right]$. In this calculation, four material constants are selected according to the previous paper as follows; $G=29.4$ $\mathrm{GPa}, \tau_{0}=79.3 \mathrm{MPa}, H=4.9 \times 10^{2} \mathrm{MPa}, \lambda$ $=2$ (Lin's assumption). The first example of two-segment strain path has a corner

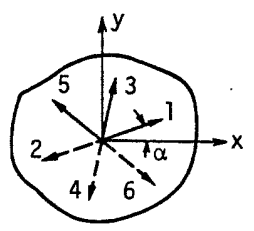

(a)

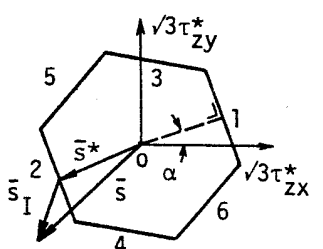

(b)
Fig. 1 Two dimensional model of single crystal and its yield surface

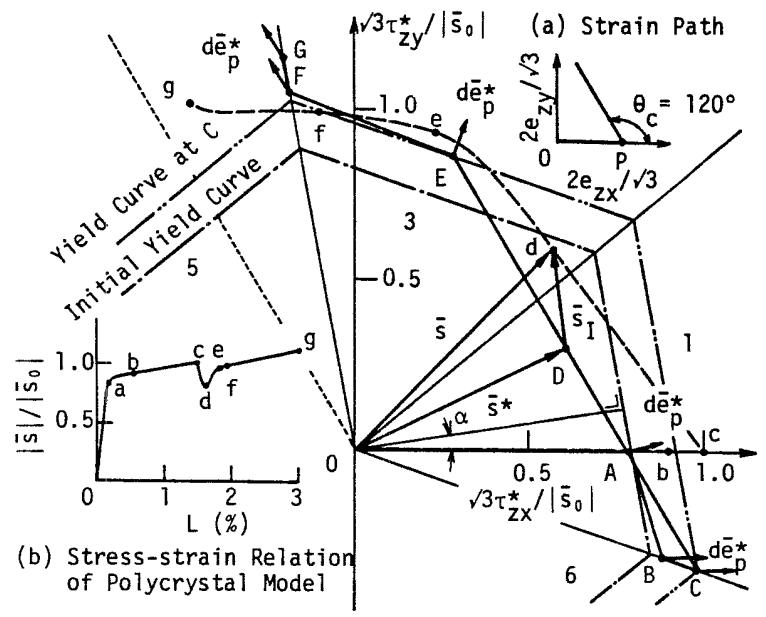

Fig. 2 Microscopic stress vector $\overline{\mathbf{s}}^{*}$ in a grain with $\alpha=10^{\circ}$ for twosegment strain path with corner angle $\theta_{c}=120^{\circ}$ angle $\theta_{c}=120^{\circ}$ and a pre-strain $L_{0}(=$ $O P)=1.5 \%$ as illustrated in the small figure (a) inserted in Fig. 2.

Figure 2 shows a variation of microscopic stress $\bar{s}^{*}=\left(\sqrt{3} \tau_{z x}^{*}, \sqrt{3} \tau_{z y}^{*}\right)$ (solid curve OABCDEFG) in a grain with an orientation $a=10^{\circ}$ in the polycrystal deformed along the strain path mentioned above. In this figure, a single-dot chained curve and a double-dot chained curve show an initial yield locus and a subsequent yield locus at the corner point $P$ of the strain path (see the inserted figure (a)), of the grain. A dashed curve $O(a) b c d e f g$ shows a variation of macroscopic (averaged) stress vector $\bar{s}=\left(\sqrt{3} \tau_{z x}, \sqrt{3} \tau_{z y}\right)$ of the polycrystal model, where the point a is omitted in the figure because it is very close to the point $A$. The representative points A, B, ..., G on the path of $\bar{s}^{*}$ (solid curve) correspond to the points a, $b, \ldots, g$ on the path of $\bar{s}$ (dashed curve). For example, the point $c$ on dashed curve shows a macroscopic stress state of polycrystal at the corner point $P$ of the strain path, and the point $C$ on the solid curve shows a microscopic stress state of single crystal with an orientation $\alpha=10^{\circ}$ at the corner point $\mathrm{P}$ of the strain path. The figure (b) inserted in Fig. 2 shows a relation between the magnitude of stress vector $|\vec{s}|=\left[\left(\sqrt{3} \tau_{z x}\right)^{2}+\left(\sqrt{3} \tau_{z y}\right)^{2}\right]^{1 / 2}$ and the length of strain path $L=$ $\int_{0}\left[\left(2 d e_{z x} / \sqrt{3}\right)^{2}+\left(2 d e_{z y} / \sqrt{3}\right)^{2}\right]^{1 / 2}$, where the points $a, b, \ldots$, g on the curve correspond to the points $A, B, \ldots, G$ on the stress path mentioned above, respectively. The parameter $\left|\vec{s}_{0}\right|$ used in the figure is a magnitude of stress vector $\bar{s}$ at the corner point $\mathrm{P}\left(\left|\bar{s}_{0}\right|=180.2 \mathrm{MPa}\right)$.

As found from the figure, the microscopic stress $\vec{s}^{*}$ increases along the $\sqrt{3} \tau_{z x}^{*}$ axis starting from the origin 0 . When $s^{*}$ reaches the point $A$ which is an intersecting point of the $\sqrt{3} \tau_{z x}^{*}$-axis and the initial yield locus (a single-dot chained curve) a slip starts to occur in the slip system 1. In the figure, directions of plastic strain increment vectors $d \bar{e}_{p}^{*}=$ $\left(2 d e_{z x}^{* P} / \sqrt{3}, 2 d e_{z y}^{* p} / \sqrt{3}\right)$ are shown by some small segments with arrow, by establishing local planes $\left(2 d e_{z x}^{* p} / \sqrt{3}, 2 d e_{z y}^{* p} / \sqrt{3}\right)$ at some typical points. The vector $d \bar{e}_{p}^{*}$ at the point $A$ is normal to the initial yield locus (singledot chained curve). The internal stress $\bar{s}_{l}$ is still very small in this stage because

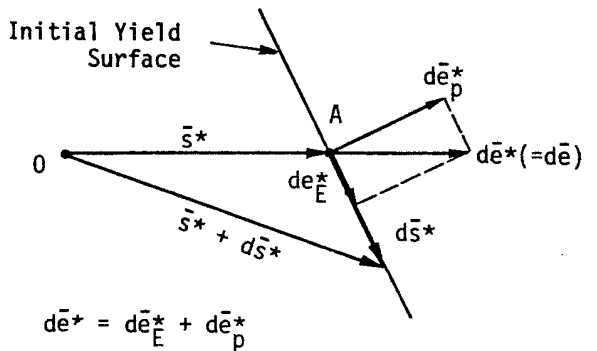

Fig. 3 Variation in direction of $\bar{s}^{*}$ after yielding 
of $\vec{s}^{*} \doteq \bar{s}$. After the point A, the vector $\bar{s}^{*}$ begins to depart from the $\sqrt{3} \tau_{z x}^{*}-$ axis. This movement can be understood by considering that $d \bar{e}_{p}^{*}$ is normal to the yield surface, $\quad d \bar{s}^{*}=2 G d \bar{e}_{E}^{*}=2 G\left[d \bar{e}^{*}-d \bar{e}_{p}^{*}\right]$ and $d \bar{e}^{*}=d \bar{e}$ (Lin!s strain constant assumption). Figuer 3 illustrates schematically geometrical relations among these vectors by assuming no work-hardening for simplicity, where $d \bar{e}_{E}^{*}$ is an elastic part of $d \bar{e}^{*}$ and thus $d \bar{e}^{*}=d \bar{e}_{E}^{*}+d \bar{e}_{p}^{*}$. When the vector $\bar{s}$ arrives at the point $B$ the slip system 6 as well as the system 1 becomes active and a so-called double-slip state appears. As found from the inserted figure (b), the point $\mathrm{B}$ corresponds to $L \fallingdotseq 0.5 \%$. In this stage, the internal stress $\bar{s}_{I}$ described in terms of a vector $\overrightarrow{B b}$ connecting the points $B$ and $b$ is a pretty big magnitude. The double-slip state continues from the point $B$ to the point $C$. The internal stress $\bar{s}_{t}$ at the point $\mathrm{C}$ is given by the vector $\overrightarrow{C C}$. As found from the figure, the averaged increasing rate of internal stress from the points $B$ to $C[=$ $\left.|\overrightarrow{C C}-\overrightarrow{B b}| / L_{(B C)}\right]$ is much smaller than that from the points $A$ to $B\left[\fallingdotseq|\overrightarrow{B b}| / L_{(A B)}\right]$, where $L_{(A B)}(\fallingdotseq 0.40 \%)$ and $L_{(B C)}(\fallingdotseq 1.05 \%)$ are lengths of strain path between the points $A$ and $B$, and between the points $B$ and $C$, respectively. That is, the double-

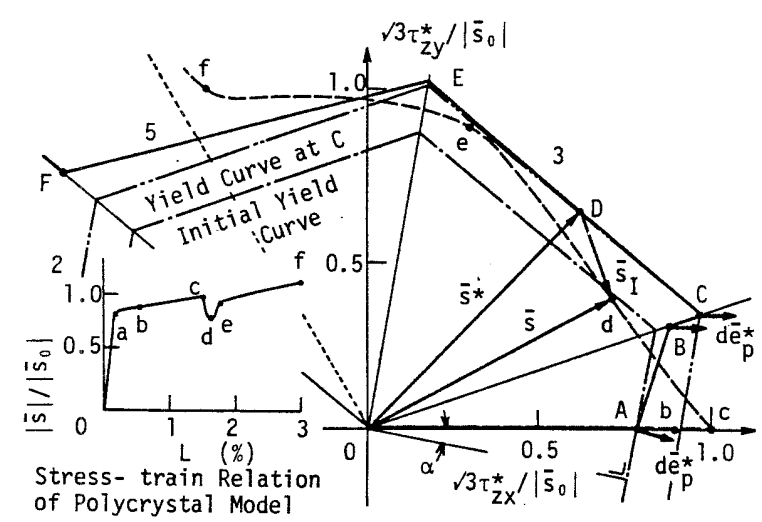

Fig. 4 Microscopic stress vector $\bar{s}^{*}$ in a grain with $\alpha=-10^{\circ}$ for twosegment strain path with corner angle $\theta_{c}=120^{\circ}$

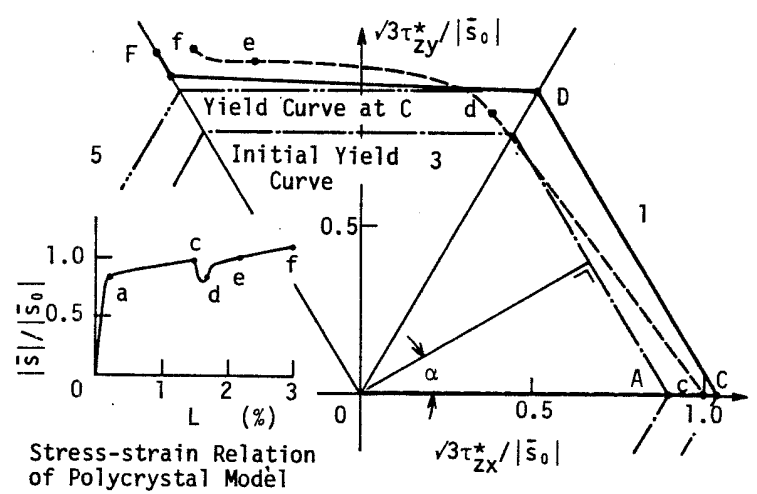

Fig. 5 Microscopic stress vector $\bar{s}^{*}$ in a grain with $\alpha=30^{\circ}$ for twosegment strain path with corner angle $\theta_{c}=120^{\circ}$ slip obstructs strongly the increase of internal stress, as suggested in the previous paper. This mechanism may be related with saturation of induced plastic anisotropy"!. Just after the corner point $P$ of strain path, the grain departs from the double-slip state and gets unloaded. The crystal grain deforms elastically till $\bar{s}^{*}$ reaches the point E. An angle between the path $\mathrm{CE}$ and the $\sqrt{3} \pi^{*} x-$ axis is $120^{\circ}$ which is the same as the corner angle of strain path. After the vector $\vec{s}^{*}$ arrives at the point $\mathrm{E}$, the slip system 3 yields a plastic strain, and after the point $F$, a double-slip state (of the systems 3 and 5) appears again, and the vector $\bar{s}^{*}$ reaches the point $G$ without any change of the direction.

Figure 4 shows a stress path $\bar{s}^{*}$ of a grain with $a=-10^{\circ}$ in the same manner as Fig. 2. This grain has an initial yield locus which is a mirror image of that of the grain with $\alpha=10^{\circ}$ in Fig. 2, with respect to the $\sqrt{3} \tau_{z x}^{*}$-axis. As found from the figure, the stress path from the origin 0 to the point $C$ is also a complete mirror image of that in Fig. 2, with respect to the $\sqrt{3} \tau_{2 x}^{*}$-axis. After the corner point $P$ of strain path, $\bar{s}^{*}$ departs from the point $C$, but it is still kept at a current yield locus. A slip occurs in the system 3 during the process that $\bar{s}^{*}$ moves from the point $C$ to the point $E$. That is, an unloading after the corner point of strain path does not occur in this grain. The slip proceeds in the system 5 from the point $\mathrm{E}$ to the point $\mathrm{F}$. At the point $F$, a double-slip state (of systems 5 and 2) appears again, and the state is kept after that. As found from the comparison of Figs. 2 and 4, two stress vectors $\bar{s}^{*}\left(\alpha=10^{\circ}\right)$ and $\bar{s}^{*}(\alpha=$ $\left.-10^{\circ}\right)$, and two plastic strain-increment vectors $d \bar{e}_{p}^{*}\left(\alpha=10^{\circ}\right)$ and $d \bar{e}_{p}^{*}\left(\alpha=-10^{\circ}\right)$ (hereafter, " $\alpha="$ is omitted) at the same instant are kept in a mirror image of each other, with respect to the $\sqrt{3} \tau_{z x}^{*}$-axis, before the corner point $P$ of strain path. That is, vectors $\bar{s}^{*}\left(10^{\circ}\right)+\bar{s}^{*}\left(-10^{\circ}\right)$ and $d \bar{e}_{p}^{*}\left(10^{\circ}\right)+d \bar{e}_{p}^{*}\left(-10^{\circ}\right)$ have zero components along the $\sqrt{3} \tau_{z x}^{*}$-axis. This means that two summation vectors are co-axial. By considering such situations over all grains in the polycrystal model, we can get a coaxiality of two macroscopic (averaged) vectors $\bar{s}$ and $d \bar{e}^{p}$ obtained by averaging $\bar{s}^{*}$ and $d \vec{e}_{p}^{*}$, respectively. On the other hand, the mirror image relations between $\bar{s}^{*}\left(10^{\circ}\right)$ and $\bar{s}^{*}\left(-10^{\circ}\right)$, between $d \bar{e}_{p}^{*}\left(10^{\circ}\right)$ and $d \bar{e}_{p}^{*}(-$ $\left.10^{\circ}\right)$ are disturbed after the corner point P. This fact explains that the direction of stress vector $\bar{s}$ does not coincide with that of plastic strain increment vector $d \bar{e}_{p}$ after the corner point $P$. In both figures, the directions of stress vectors $\bar{s}^{*}$ do not change after the point $F$ at which the double-slip states appear. These states are similar to those obtained in a deformation test of the polycrystal model along a straight strain path which emanates from the origin in the direction of the angle $120^{\circ}$ from the $\sqrt{3} \tau_{z x}^{*}$-axis as shown by the dotted line. This fact tells that the co-axiality of macroscopic stress $\bar{s}$ and macroscopic 
plastic strain increment $d \bar{e}_{p}^{-}$appears again after some increase of deformation along the straight segmen as observed in the experiments. ${ }^{119}$

Figure 5 shows a variation of $\vec{s}^{*}$ of a grain with an orientaion $\alpha=30^{\circ}$ in the polycrystal model deformed along the twosegment strain path. In this case, the stress state is kept in neutral loading just after the corner point $P$ of strain path (see the microscopic stress path $\mathrm{C} \rightarrow$ D). On the other hand, the stress states just after the corner point $P$ in the grains with orientations $\alpha=10^{\circ}$ and $-10^{\circ}$ are in unloading and in loading, respectively. That is, it is likely that the deformation is macroscopically plastic just after the corner even if the corner angle of strain path is larger than $90^{\circ}$, but microscopically some grains are in loading, some are in unloading and others are in neutral.

Figures 6 and 7 show the stress paths

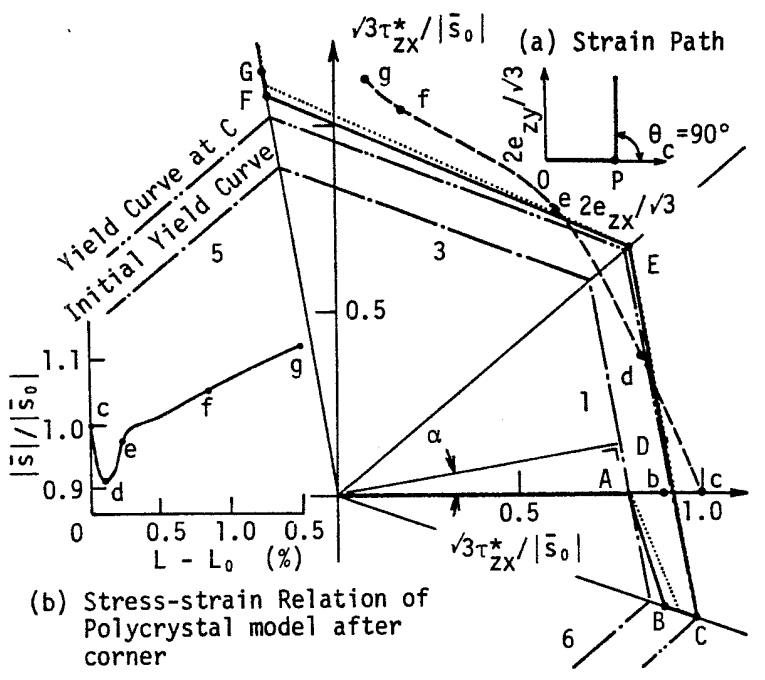

Fig. 6 Microscopic stress vector $\bar{s}^{*}$ in a grain with $\alpha=10^{\circ}$ for twosegment strain path with corner angle $\theta_{\mathrm{c}}=90^{\circ}$

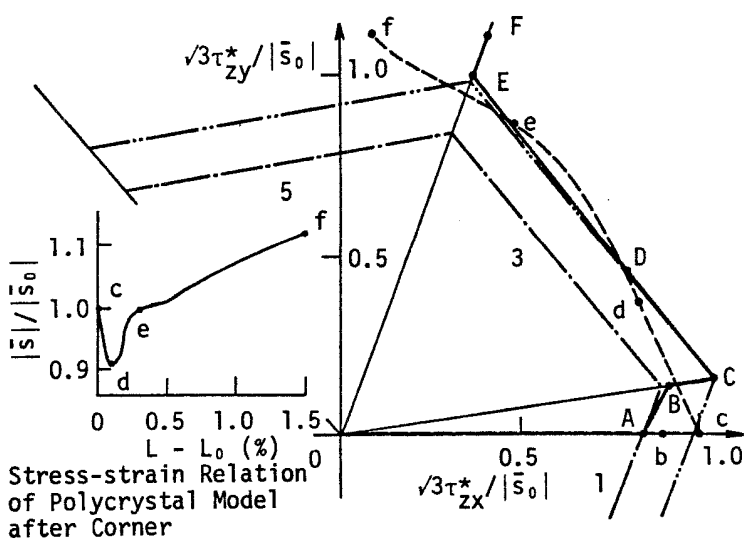

Fig. 7 Microscopic stress vector $\bar{s}^{*}$ in a grain with $\alpha=-20^{\circ}$ for twosegment strain path with corner angle $\theta_{c}=90^{\circ}$ of grains with orientations $\alpha=10^{\circ}$ and $-10^{\circ}$ in the polycrystal model deformed along a two-segment strain path with a right-angle corner shown in a inserted Fig. (a) in Fig. 6. We can guess easily from these figures that all grains (imagine the grains with $-30^{\circ}<\alpha \leqq 30^{\circ}$ ) keep themselves loaded just after the corner point $P$ of strain path. The inserted figure (b) in Fig. 6 (or Fig. 7) show the macroscopic stress-strain relation after the corner point. As found from the inserted figure, the magnitude of stress vector $\left|\vec{s}^{*}\right|$ drops temporarily just after the corner point $P$, although all grains are kept in loading states. This stress-drop has been also confirmed experimentally! This phenomenon seems to be related with the double-slip and the shape of yield locus. Geometrically, the corner point $C$ of yield locus is farther apart from the origin 0 than any other point on the yield locus. This geometrical situation shows that the magnitude of stress vector just after the corner becomes smaller temporarily than that on the corner point C. The dotted line in Fig. 6 shows a variation of $\bar{s}^{*}$ obtained by using Kroner's self-consistent assumption $(\nu=$ 0.3). As found from the comparison of solid and dotted curves, there may be no substantial difference between the two assumpstions in the cases discussed here.

\section{Fundamental Micro-mechanics of Mechanical Ratcheting Phenomenon and Multi-slip}

4.1 Initiation and development of ratcheting strain

The initation and development of ratcheting strain may be closely related with the mechanical properties of single crystal component, especially the discrete distribution of slip systems and the double-slip caused by the increased internal stress. When a constant shear stress $\tau_{z y}$ (as a stationary stress) as well as a cyclically changing shear strain $e_{z x}$ is applied to the two-dimensional polycrystal model explained in Section 2, a permanent strain $e_{z y}^{p}$ (that is, ratcheting strain) may be accumulated in each cycle. This type of mechanical ratcheting phenomenon is here analyzed and discussed from a micro-mechanics point of view. In our analysis, it is assumed that the single crystal component does not have any strain hardening $[\mathrm{H}=0$ in Eq. (1)]. This assumption may be reasonable to get fundamental micro-mechanisms qualitatively when we note that hysteresis loops (for example, $\sigma_{\mathbf{z}}-\varepsilon_{\mathbf{z}}$ curve obtained by a cycle tension-compression test) are stable after a comparatively small number of cycles. Kröner's self-consistent assumption is used to incorporate the interactions among grains in the polycrystal. Then $\nu=0.3$ is selected in Eq. (3). Other two material constants $G$ and $\tau_{0}$ are the same as those used in the previous section.

Now, let's discuss mechanical behaviours of typical single crystal components in the polycrystal model subjected to, for example, a stationary stress $\sigma(=$ 
$\left.\sqrt{3} \tau_{\mathrm{ay}}\right)=38.2 \mathrm{MPa}$ and a strain amplitude $a\left(=2 e_{z r} / \sqrt{3}\right)= \pm 2 \%$, based on the computationally analyzed results.

Figure 8 shows a variation of stress $\bar{s}^{*}$ and some strain increments $d \bar{e}_{p}^{*}$ appearing in a single crystal component with an orientation $\alpha=30^{\circ}$ in the polycrystal model. The stress vector $\vec{s}^{*}$ moves on $0 \rightarrow$ A, with application of stationary stress. The value of $O A$ equals to the stationary stress $\sigma\left(=\sqrt{3} \tau_{2 x}=38.2 \mathrm{MPa}\right)$ because every grain is deformed elastically at this level of stress. When the cyclic macroscopic straining whose amplitude is $a$ (= $\left.2 e_{z z} / \sqrt{3}\right)= \pm 2 \%$ is added to the polycrystal model while the stationary stress is kept,

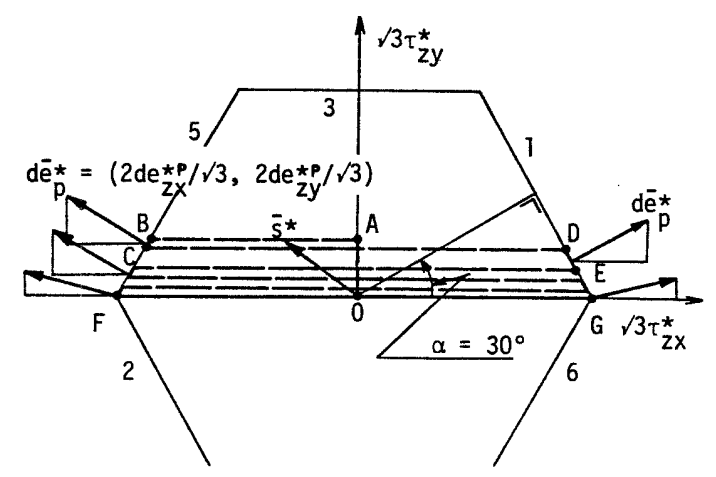

Fig. $8 \bar{s}^{*}$ and $\overline{d e}^{*}$ in a grain with $\alpha$ $=30^{\circ}$ in mechanical ratcheting deformation process

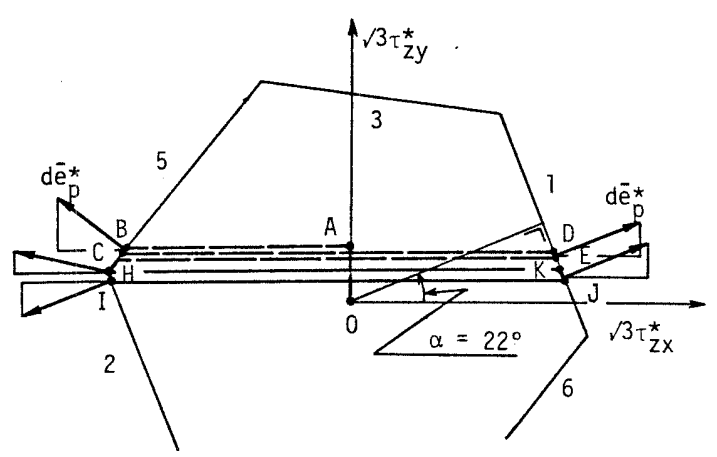

Fig. $9 \bar{s}^{*}$ and $\bar{e}_{\hat{p}}^{*}$ in a grain with $\alpha$ $=22^{\circ}$ in mechanical ratcheting deformation process

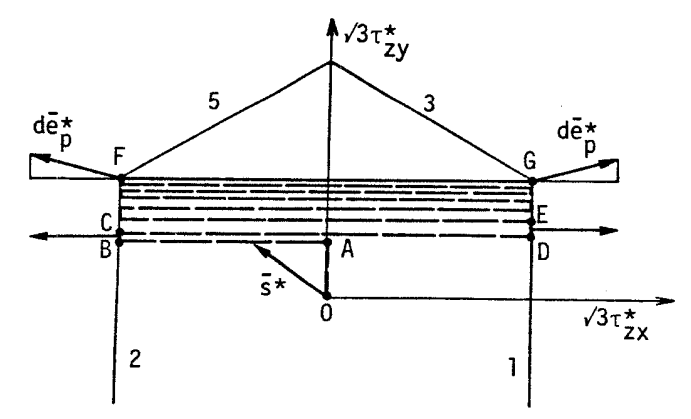

Fig. $10 \bar{s}^{*}$ and $d^{-}{ }^{*}$ in a grain with $\alpha$ $=0^{\circ}$ in mechanical ratcheting deformation process $\vec{s}^{*}$ in the single crystal moves along the dashed curve $A \rightarrow B \rightarrow C \rightarrow \ldots$, as shown in Fig. 8. When the stress $\vec{s}^{*}$ moves on the yield locus $(B \leftarrow C, D \bullet E$, etc), the plastic strain increment vector $d \bar{e}_{p}^{*}$ occurs in the normal direction to the yield locus. Then, the component de $e_{z y}^{*}$ of $d \bar{e}_{p}^{*}$ is accumulated and becomes a part of the macroscopic ratcheting strain. After the stress vector $\vec{s}$ reaches the point $F$ (double-slip of systems 5 and 2 at the point F), the stress vector $\bar{s}^{*}$ moves cyclically between the point $F$ and the point $G$ (solid line). In this stage, the plastic strain increments $d \bar{e}_{p}^{*}$ are produced in the directions as shown by the small segments with arrow, at the points $F$ and G. Thus, the component $d e_{z y}^{* p}$ of $d \bar{e}_{p}^{*}$ is accumulated as the ratcheting strain. As found from this mechanism, the rate of microscopic ratcheting strain per cycle in this grain may become constant after $\vec{s}^{*}$ reaches the point $F_{\text {. }}$

Figure 9 shows a case of a single crystal component with $\alpha=22^{\circ}$. The vector $\vec{s}^{*}$. in the grain moves on the dashed curve $(0 \rightarrow A \rightarrow B \rightarrow C \rightarrow D \rightarrow E)$ and produces the ratcheting component on $\mathrm{B} \rightarrow \mathrm{C}$ and $\mathrm{D} \rightarrow \mathrm{E}$ ( $d e_{z y}^{* p}$ of $d \bar{e}_{p}^{*}$ ). A part of movement of $\vec{s}^{*}$ is omitted in order to avoid a crowding of lines. After a specific number of cycles, the vector $\bar{s}^{*}$ moves cyclically on the closed solid curve $(H \rightarrow K \rightarrow J \rightarrow I \rightarrow H)$. As found from the figure, when the vector $\bar{s}^{*}$ moves on $\mathrm{I} \rightarrow \mathrm{H}$, the component de $e_{z y}^{* p}$ is negative, and when the vector $\vec{s}^{*}$ moves on $\mathrm{K} \rightarrow \mathrm{J}$, the component de $e_{y}^{* p}$ is positive. As the result, $\int^{H} d e_{z y}^{* p}+\int_{x}^{J} d e_{z y}^{* p}+\int_{H} d e_{z y}^{* p}>0$ is accumulated in each cycle, where $\int_{t}^{*} e_{z y}^{* p}$ is an accumulated component during the state that $\bar{s}^{*}$ stays at the point $\mathrm{H}$.

Figure 10 shows a case of a single crystal component with $\alpha=0$ : In this case, after a few cycles, double-slip states occur at the points $F$ and $G$. Then, $\bar{s}^{*}$ moves cyclically on the solid line $\mathrm{F}$. $G$, and producdes the ratcheting component de $e_{z y}^{* p}$ at the points $F$ and $G$.

As seen from the above discussions on the computational results, there are two stages in the process; the first stage in which the microscopic stress $\tilde{s}^{*}$ makes a transient movement and the second stage in which $\vec{s}^{*}$ moves cyclically on a closed circuit in the presence of double-slip. In accordance with the movement of $\bar{s}^{*}$, there may be two stages in the rate of a macroscopic ratcheting strain. Especial$1 y$, the rate of macroscopic ratcheting strain (per cycle) may be constant in the second stage.

Figure 11 shows variations of macroscopic ratcheting strains $\left(2 e_{z}^{D} / \sqrt{3}\right)$ obtained by averaging such.microscopic ratcheting strains over all single crystal components in the polycrystal model. These computational results reproduce fundamental features of mechanical ratcheting phenomenon observed in the experiments.

As seen from the above discussions, the fundamental mechanism of ratcheting phenomenon is closely related with the occurrence of multi-slip due to the increased internal stress. 


\subsection{Recovery of ratcheting strain}

It was observed experimentally that an accumulated ratcheting strain decreases with a continued cyclic straining after a steady stress is lifted. This phenomenon is called a recovery of ratcheting strain. In order to investigate fundamental mechanisms of ratcheting recovery, the following series of loading processes are considered;

(1) 10 cycles of an amplitude $a=$ $\left(2 e_{z x} / \sqrt{3}\right)= \pm 0.2 \%$ with a steady stress $\sigma=$ $\left(\sqrt{3} \tau_{z y}\right)=38.2 \mathrm{MPa}$.

(2) The steady stress is lifted.

(3) Cyclic straining with the same amplitude as that in (1) without the steady stress.

In the same manner as in the previous section, stresses $\vec{s}^{*}$ and plastic strain increments $d \bar{e}_{p}^{*}$ in some typical single crystal components are discussed on the basis of computational results.

Figure 12 shows $\bar{s}^{*}$ and $\bar{e}_{p}^{*}$ in single crystal components with an orientation $\alpha=30^{\circ}$ in the polycrystal model. The vector $\vec{s}^{*}$ in the process (1) moves cyclically on $F \leftrightarrow G$ and at the end of the process $(1)\left(2 e_{z x} / \sqrt{3}=0\right), \bar{s}^{*}$ is on the point $0^{\prime}$. In the process (2), $\vec{s}^{*}$ moves elastically from the point $O^{\prime}$ to the point $A$. By the cyclic straining without steady stress, that is, in the process (3), the vector $\vec{s}^{*}$ moves on the dashed curve $A \rightarrow B \rightarrow C \rightarrow$ $\mathrm{D} \rightarrow \mathrm{E} \rightarrow \ldots$. In the process, when the vector $\bar{s}^{*}$ is on the yield locus $(B \rightarrow C, D \rightarrow E$, etc), the plastic strain increment vector $d \vec{e}_{p}^{*}$ is produced and the component $d e_{z y}^{* p}<0 \mathrm{re}-$ duces the ratcheting strain accumulated in the process (1). After the vector $\bar{s}^{*}$ reaches the point $F, \bar{s}^{*}$ moves again cyclically on the line $F \leftrightarrow G$. In the first

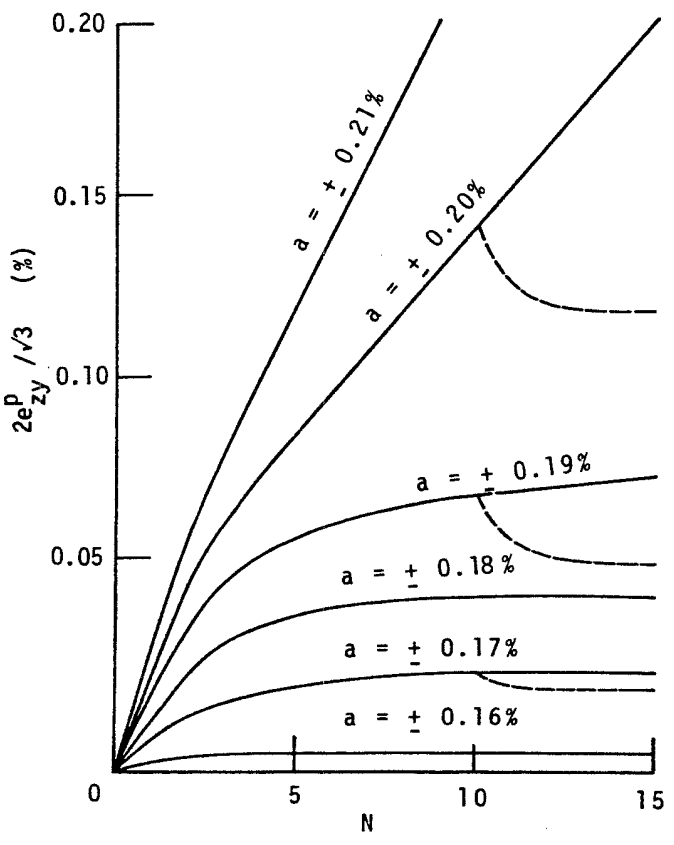

Fig. 11 Development and recovery of mechanical ratcheting strain few cycles of such movement, $\vec{s}^{*}$ produces $d \bar{e}_{p}^{*}$ at the points $F$ and $G$, in the directions shown by dashed segments with arrow as illustrated in Fig. 12. The directions of $d \bar{e}_{p}^{*}$ rotate to the directions shown by the solid segments with arrow and then the recovery of ratcheting strain is finished in this grain.

Figure 13 shows a case of a grain with $\alpha=28^{\circ}$. The stress $\bar{s}^{*}$ in this grain moves along the dashed curve $A \rightarrow B \rightarrow C \rightarrow$ ... When $\vec{s}^{*}$ moves on the yield surface, $d \bar{e}_{p}^{*}$ is produced and the component $d e_{z j}^{* p}<0$ reduces the accumulated ratcheting strain. After a few cycles, $\vec{s}^{*}$ circulates in the closed path $(H \rightarrow I \rightarrow J \rightarrow K \rightarrow H)$. The ratcheting

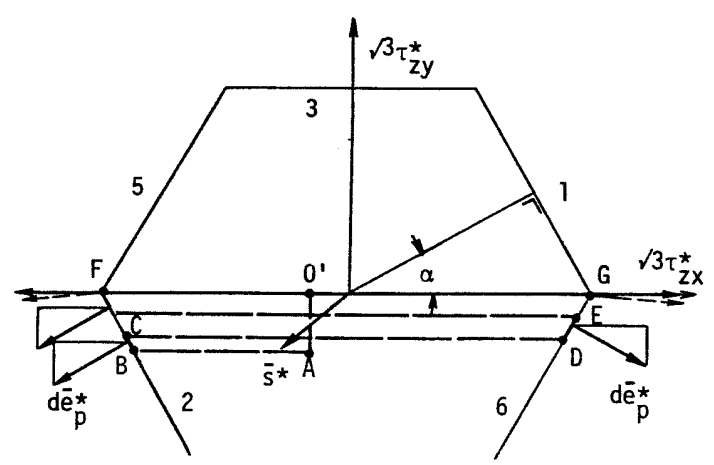

Fig. $12 \bar{s}^{*}$ and de $\bar{e}^{*}$ in a grain with $\alpha=$ $30^{\circ}$ during recovery process of mechanical ratcheting strain

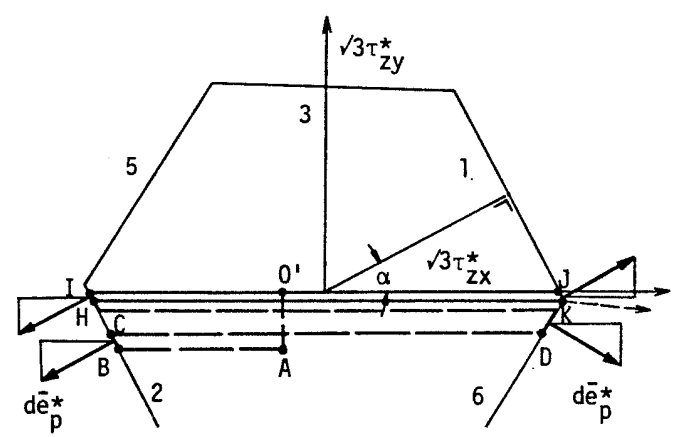

Fig. $13 \bar{s}^{*}$ and $\bar{e}^{*}$ in a grain with $\alpha=$ $28^{\circ}$ during recovery process of mechanical ratcheting strain

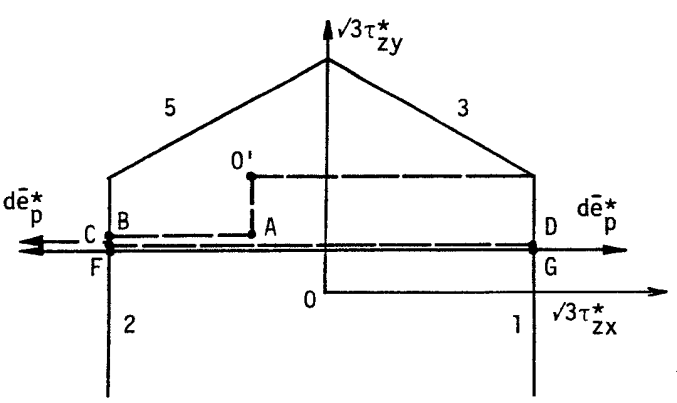

Fig. $14 \bar{s}^{*}$ and $d \bar{e}^{*}$ in a grain with $\alpha=$ $0^{\circ}$ during recovery process of mechanical ratcheting strain 
strain is reduced for a reason just opposite to the reason about the closed path $\mathrm{H} \rightarrow$ $I \rightarrow J \rightarrow K \rightarrow H$ shown in Fig. 9. According to some detailed checking of computational results, after a specific number of cycles, the double-slip of systems 1 and 6 on the point $K$ disappears and the vector $\vec{s}^{*}$ does not stay on the point $K$ though $\vec{s}$ goes on past the point $K$. Roughly speaking, in this stage, the recovery of ratcheting strain is finished in this grain. Figure 14 shows a case of a grain with $\alpha=0^{\circ}$. As found from the figure, this grain does not make any direct contribution to the recovery. This grain keeps the value of $\sqrt{3} \tau_{z y}^{*}$ to make the value of steady stress $\sqrt{3} \tau_{z y}$ zero in the polycrystal.

The dashed curve in Fig. 11 shows the (macroscopic) recovery of ratcheting strain obtained by averaging such microscopic recoveries of ratcheting strains over all grains in the polycrystal model, as well as other cases ( $a= \pm 0.19 \%$, $\pm 0.17 \%)$. As found from this figure, the computational analysis reproduces fundamental features of recovery in the mechanical ratcheting strain observed in the experiments.

\section{Conclusions}

In this paper, internal stresses and multi-slips in single crystal components of polycrystal were studied by using a simple mechanical model of polycrystal, in two typical examples of complex loadings, and these microscopic mechanisms were confirmed to be closely related with typical macroscopic features of plastic behaviour of polycrystal observed in the experiments.

In the first example, that is, in stress responses for two-segment strain path, it was shown that the multi-slip prevents an increase of internal stress and thus prevents a rupture of material in the earlier stage of deformation. The multi-slip plays also an important role in the phenomenon that the difference between the directions of stress vector and plastic strain increment vector disappears after some increase of deformation along straight segment of strain path. This phenomenon is one aspect of "fading memory" on the preceding deformation history. Moreover, a discrete distribution of slip systems in the grain explains why the magnitude of stress vector drops just after the corner temporarily while the polycrystal yields a plastic deformation during the process.

In the second example, that is, in mechanical ratcheting phenomenon, it was shown that two typical increase modes (transient and steady) of ratcheting strain are closely related with the double-slip mechanism in the single crystal components. Also, the recovery of ratcheting strain can be understood as a transient process from the single slip to the double slip in the grain.

Micro-mechanisms of polycrystalline metals are much more complex than those discussed here, and more than two slip systems become active simulteneously. ${ }^{[23]}$ For example in the ratcheting phenomenon, dislocation structures in single crystal components change in complex manner under cyclic loading and thus the yield surface of each single crystal component shows a complex change in its shape and position. Therefore, in order to formulate a set of practical constitutive equations based on the model proposed here, it may be necessary to incorporate such complex changes of yield surfaces. Neverthless it should be noted that in plastic deformations of polycrystals, internal stresses caused by interactions among grains and double slips closely related with the increase of internal stress play a very important role and there are several interesting phenomena which we can not evaluate without such knowledges of the micro-mechanism.

\section{References}

[1] Toduda, M. et al, Bulletin of the JSME, 25-208(1982), 1491

[2] Abe, T., J. Soc. Mate. Sci., Japan, 28-305(1979), 1 (in Japanese)

[3] Kocks, K.F., Metall. Trans.,1(1970), 1.121

[4] Asaro, R.J., Advances in Applied Mechanics (eds., Hutchinson, J.W. and Wu, T.Y.),23(1983),1, Academic Press

[5] Havner, K.S., Mechanics of Solids ; The Rodney Hill 60th Aniversary Volume (eds. Hopkins, H.G. and Swell, M.J.) (1982), 265, pergamon Press Ltd.

[6] Udoguchi, E., et al., Trans. of the JSME, 42-395(1976), 1982, 1993, (in Japanese)

[7] Tanaka, K., et al., Trans. of the JSME, 42-358(1976), 1685(in Japanese)

[8]. Yoshida, S., et al., Trans. of the JSME, 46-409(1980),799(in Japanese)

[9] Benham, P.P., Int. J. Mech. Sci., 7 (1965), 81

[10] Tokuda, M. , et al., Proceedings of the JSME, 843-1(1984), 66(in Japanese)

[11] Kaneko, K., Trans. of the JSME, 46-405(1980), 505(in Japanese)

[12] Yoshida, S., Trans. of the JSME, 43-371(1977), 2501 (in Japanese)

[13] Ohashi, Y., Trans. of the JSME. 48-428(1982), 389(in Japanese)

[14] Taylor, G.I., Proc. Roy. Soc. Lond., 116A(1927), 39

[15] Hutchinson, J.W., J. Mech. Phys. Solids, 12(1964), 11

[16] Kröner, E., Acta Meta., 9(1961), 115

[17] Ilyushin, A.A., Plasticity, Foundation of General Plastic Theory, (1963), 26, Izd. Akad. Nauk., USSR, Moscow

[18] Ohashi, Y., The Science of Machine, 23-2(1972) 343;23-3(1972),479; 234(1972),611 (in Japanese)

[19] Ohashi, Y., et al., J. Mech. Phys. Solids, 23(1975), 295

[20] Lin, T.H., J. Mech. Phys. Solids, $5(1957), 143$

[21] Ohashi, Y. and Tokuda, M., J. Mech. Phys. Solids, 21(1973), 241

[22] Gotoh, M., Trans. of the JSME, 45-396(1980), 943(in Japanese) 